\title{
Changing-periodic time scales and decomposition theorems of time scales with applications to functions with local almost periodicity and automorphy
}

\author{
Chao Wang ${ }^{1}$ and Ravi P Agarwal $2,3^{*}$
}

"Correspondence:

Agarwal@tamuk.edu

${ }^{2}$ Department of Mathematics, Texas

A\&M University-Kingsville,

700 University Blvd., Kingsville,

TX 78363-8202, USA

${ }^{3}$ Department of Mathematics,

Faculty of Science, King Abdulaziz

University, P.O. Box 80203, Jeddah,

21589, Saudi Arabia

Full list of author information is

available at the end of the article

\begin{abstract}
In this work, we address the periodic coverage phenomenon on arbitrary unbounded time scales and initiate a new idea, namely, we introduce the concept of changing-periodic time scales. We discuss some properties of this new concept and illustrate several examples. Specially, we establish a basic decomposition theorem of time scales which provides bridges between periodic time scales and an arbitrary time scale with a bounded graininess function $\mu$. Based on this result, we introduce local-almost periodic and local-almost automorphic functions on changing-periodic time scales and study some related properties. The concept of changing-periodic time scales introduced in this paper will help in understanding and removing the serious deficiency which arises in the study of classical functions on time scales.
\end{abstract}

MSC: 26E70; 43A60; 26E99; 34N05; 35B15

Keywords: changing-periodic time scales; decomposition theorem of time scales; periodic sub-timescale; almost periodic functions; almost automorphic functions

\section{Introduction}

With the development and deepening of the real analysis on time scales, many works related to time scales have appeared recently (see [1-11]). This has resulted in the study of dynamic equations on time scales becoming a leading topic of research. Specially, to study periodic solutions of dynamic equations on time scales, Kaufmann and Raffoul in 2006 (see [12]) introduced the concepts of periodic time scales and periodic functions. This was followed (see $[13,14]$ ) by the definitions of almost periodic time scales and almost periodic functions to investigate almost periodic solutions of dynamic equations on time scales. We recall that a time scale is an irregular closed subset of $\mathbb{R}$, and thus it creates several difficulties of keeping the closedness of variables of functions under translation; for example, for a given function $f: \mathbb{T} \rightarrow \mathbb{R}$, we cannot guarantee that there exists $\tau$ such that $f(t+\tau)$ makes sense because we do not know whether $t+\tau \in \mathbb{T}$. This is one of the main reasons mathematicians have introduced some suitable time scales such as periodic time scales and almost periodic time scales, and so on, before even they give the proper concepts of functions defined on time scales.

(c) 2015 Wang and Agarwal. This article is distributed under the terms of the Creative Commons Attribution 4.0 International License (http://creativecommons.org/licenses/by/4.0/), which permits unrestricted use, distribution, and reproduction in any medium, provided you give appropriate credit to the original author(s) and the source, provide a link to the Creative Commons license, and indicate if changes were made. 
It is clear that periodic time scales are regular, so we can obtain some of their nice properties (such as the above closedness property), and introduce and study well-defined functions on them. However, most of time scales are not periodic; thus, it is necessary and meaningful to find an effective way to build bridges between periodic time scales and an arbitrary time scale. This will easily allow us to extract results known for periodic time scales to other types of time scales, and vice versa. Having this in mind, in this paper, we shall introduce a new concept, which we call 'changing-periodic time scales'. This concept deals not only with almost periodic time scales, but also with all the time scales with the bounded graininess function $\mu$. In conclusion, we shall show that all the time scales with a bounded graininess function $\mu$ can be decomposed into a countable union of periodic time scales, i.e., we shall formulate a basic decomposition theorem of time scales. This result paves a way to study functions on an arbitrary time scale with bounded $\mu$ as conveniently as on periodic time scales. Next, based on changing-periodic time scales, we shall introduce 'local-almost periodic' and 'local-almost automorphic' functions and study some of their related properties. Finally, we shall apply these results to address local-almost periodicity and local-almost automorphy of solutions of dynamic equations on arbitrary time scales with bounded graininess function $\mu$.

The present paper is organized as follows. In Section 2, we begin with necessary known definitions and results on time scales, and then we introduce some new concepts, which include changing-periodic time scales. Based on these concepts, we shall establish several new results. Our main result here is the decomposition theorem of time scales. In Section 3, as applications of changing-periodic time scales, first we shall introduce two new concepts - local-almost periodic and local-almost automorphic functions, and then we show that these concepts can be used as a powerful tool to investigate the local-almost periodicity and local-almost automorphy of solutions of dynamic equations on time scales.

\section{Changing-periodic time scales}

In the following, we introduce some basic knowledge of time scales and famous Zorn's lemma which will be used in our paper. For more details, see [3-5, 15].

A time scale $\mathbb{T}$ is a closed subset of $\mathbb{R}$. It follows that the jump operators $\sigma, \varrho: \mathbb{T} \rightarrow \mathbb{T}$ defined by $\sigma(t)=\inf \{s \in \mathbb{T}: s>t\}$ and $\varrho(t)=\sup \{s \in \mathbb{T}: s<t\}$ (supplemented by inf $\phi:=$ $\sup \mathbb{T}$ and $\sup \phi:=\inf \mathbb{T}$ ) are well defined. The point $t \in \mathbb{T}$ is left-dense, left-scattered, rightdense, right-scattered if $\varrho(t)=t, \varrho(t)<t, \sigma(t)=t, \sigma(t)>t$, respectively. If $\mathbb{T}$ has a left scatter maximum $M$, define $\mathbb{T}^{k}:=\mathbb{T} \backslash M$; otherwise, set $\mathbb{T}^{k}=\mathbb{T}$. If $\mathbb{T}$ has a right scatter minimum $m$, define $\mathbb{T}_{k}:=\mathbb{T} \backslash m$; otherwise, set $\mathbb{T}_{k}=\mathbb{T}$. The notations $[a, b]_{\mathbb{T}},[a, b)_{\mathbb{T}}$ and so on will denote time scale intervals

$$
[a, b]_{\mathbb{T}}=\{t \in \mathbb{T}: a \leq t \leq b\}
$$

where $a, b \in \mathbb{T}$ with $a<\varrho(b)$.

The graininess function is defined by $v: \mathbb{T} \rightarrow[0, \infty): v(t):=t-\varrho(t)$ for all $t \in \mathbb{T}$.

The function $f: \mathbb{T} \rightarrow \mathbb{R}$ is called ld-continuous provided it is continuous at each leftdense point and has a right-sided limit at each point, we write $f \in C_{\mathrm{ld}}(\mathbb{T})=C_{\mathrm{ld}}(\mathbb{T}, \mathbb{R})$. Let $t \in \mathbb{T}_{k}$, the nabla-derivative of $f$ at $t$ denoted as $f^{\nabla}(t)$ satisfies the inequality

$$
\left|f(\varrho(t))-f(s)-f^{\nabla}(t)[\varrho(t)-s]\right| \leq \varepsilon|\varrho(t)-s|
$$


for any $\varepsilon>0$ and all $s \in U$; here $U$ is a neighborhood of $t$. Let $F$ be a function, it is called antiderivative of $f: \mathbb{T} \rightarrow \mathbb{R}$ provided

$$
F^{\nabla}(t)=f(t) \quad \text { for each } t \in \mathbb{T}_{k} \text {. }
$$

Definition 2.1 ([3-5]) A function $p: \mathbb{T} \rightarrow \mathbb{R}$ is called $v$-regressive provided $1-v(t) p(t) \neq$ 0 for all $t \in \mathbb{T}_{k}$. The set of all regressive and ld-continuous functions $p: \mathbb{T} \rightarrow \mathbb{R}$ will be denoted by $\mathcal{R}_{v}=\mathcal{R}_{v}(\mathbb{T})=\mathcal{R}_{v}(\mathbb{T}, \mathbb{R})$. We also define the set $\mathcal{R}_{v}^{+}=\mathcal{R}_{v}^{+}(\mathbb{T}, \mathbb{R})=\left\{p \in \mathcal{R}_{v}\right.$ : $1-v(t) p(t)>0, \forall t \in \mathbb{T}\}$.

The graininess function is defined by $\mu: \mathbb{T} \rightarrow[0, \infty): \mu(t):=\sigma(t)-t$ for all $t \in \mathbb{T}$.

The function $f: \mathbb{T} \rightarrow \mathbb{R}$ is called rd-continuous provided it is continuous at each rightdense point and has a left-sided limit at each point, we write $f \in C_{\mathrm{rd}}(\mathbb{T})=C_{\mathrm{rd}}(\mathbb{T}, \mathbb{R})$. Let $t \in \mathbb{T}^{k}$, the delta-derivative of $f$ at $t$ denoted as $f^{\Delta}(t)$ satisfies the inequality

$$
\left|f(\sigma(t))-f(s)-f^{\Delta}(t)[\sigma(t)-s]\right| \leq \varepsilon|\sigma(t)-s|
$$

for any $\varepsilon>0$ and all $s \in U$; here $U$ is a neighborhood of $t$. Let $F$ be a function, it is called antiderivative of $f: \mathbb{T} \rightarrow \mathbb{R}$ provided

$$
F^{\Delta}(t)=f(t) \quad \text { for each } t \in \mathbb{T}^{k}
$$

Definition 2.2([3-5]) A function $p: \mathbb{T} \rightarrow \mathbb{R}$ is called regressive provided $1+\mu(t) p(t) \neq 0$ for all $t \in \mathbb{T}^{k}$. The set of all regressive and rd-continuous functions $p: \mathbb{T} \rightarrow \mathbb{R}$ will be denoted by $\mathcal{R}=\mathcal{R}(\mathbb{T})=\mathcal{R}(\mathbb{T}, \mathbb{R})$. We also define the set $\mathcal{R}^{+}=\mathcal{R}^{+}(\mathbb{T}, \mathbb{R})=\{p \in \mathcal{R}: 1+$ $\mu(t) p(t)>0, \forall t \in \mathbb{T}\}$.

Lemma 2.1 ([15], Zorn's lemma) Suppose that $(P, \preceq)$ is a partially ordered set. A subset $T$ is totally ordered if for any $s, t$ in $T$ we have $s \preceq t$ or $t \preceq s$. Such a set $T$ has an upper bound $u$ in $P$ ift $\leq u$ for all $t$ in $T$. Suppose that a nonempty partially ordered set $P$ has the property that every nonempty chain has an upper bound in $P$. Then the set $P$ contains at least one maximal element.

In order to introduce the concept of changing-periodic time scales precisely and concisely, we need the following definitions.

Definition 2.3 We say a time scale is an infinite time scale if one of the following conditions is satisfied: $\sup \mathbb{T}=+\infty$ and $\inf \mathbb{T}=-\infty$ or $\sup \mathbb{T}=+\infty$ or inf $\mathbb{T}=-\infty$.

Definition 2.4 Let $\mathbb{T}$ be a time scale, we say $\mathbb{T}$ is a zero-periodic time scale if and only if there exists no nonzero real number $\omega$ such that $t+\omega \in \mathbb{T}$ for all $t \in \mathbb{T}$.

Remark 2.2 By Definition 2.4, it follows that a finite union of the closed intervals can be regarded as a zero-periodic time scale. The single point set $\{a\}$ can also be regarded as a closed interval since $\{a\}=[a, a]$ in this paper. For convenience, $\mathbb{T}_{0}$ denotes the zeroperiodic time scale. 
Definition 2.5 A timescale sequence $\left\{\mathbb{T}_{i}\right\}_{i \in \mathbb{Z}^{+}}$is called well-connected if and only if for $i \neq j$, we have $\mathbb{T}_{i} \cap \mathbb{T}_{j}=\left\{t_{i j}^{k}\right\}_{k \in \mathbb{Z}}$, where $\left\{t_{i j}^{k}\right\}$ is a countable points set or an empty set, and $t_{i j}^{k}$ is called the connected point between $\mathbb{T}_{i}$ and $\mathbb{T}_{j}$ for each $k \in \mathbb{Z}$, the set $\left\{t_{i j}^{k}\right\}$ is called the connected points set of this well-connected sequence.

Remark 2.3 Clearly the connected points set of a well-connected sequence can be an empty set. Hence, it follows that if $\bigcap_{i=1}^{\infty} \mathbb{T}_{i}=\emptyset$, then $\left\{\mathbb{T}_{i}\right\}_{i \in \mathbb{Z}^{+}}$is well connected.

We now introduce a new concept of time scales called 'changing-periodic time scales'.

Definition 2.6 Let $\mathbb{T}$ be an infinite time scale. We say $\mathbb{T}$ is a changing-periodic or a piecewise-periodic time scale if the following conditions are fulfilled:

(a) $\mathbb{T}=\left(\bigcup_{i=1}^{\infty} \mathbb{T}_{i}\right) \cup \mathbb{T}_{r}$ and $\left\{\mathbb{T}_{i}\right\}_{i \in \mathbb{Z}^{+}}$is a well-connected timescale sequence, where $\mathbb{T}_{r}=\bigcup_{i=1}^{k}\left[\alpha_{i}, \beta_{i}\right]$ and $k$ is some finite number, and $\left[\alpha_{i}, \beta_{i}\right]$ are closed intervals for $i=1,2, \ldots, k$ or $\mathbb{T}_{r}=\emptyset$;

(b) $S_{i}$ is a nonempty subset of $\mathbb{R}$ with $0 \notin S_{i}$ for each $i \in \mathbb{Z}^{+}$and $\Pi=\left(\bigcup_{i=1}^{\infty} S_{i}\right) \cup R_{0}$, where $R_{0}=\{0\}$ or $R_{0}=\emptyset$;

(c) for all $t \in \mathbb{T}_{i}$ and all $\omega \in S_{i}$, we have $t+\omega \in \mathbb{T}_{i}$, i.e., $\mathbb{T}_{i}$ is an $\omega$-periodic time scale;

(d) for $i \neq j$, for all $t \in \mathbb{T}_{i} \backslash\left\{t_{i j}^{k}\right\}$ and all $\omega \in S_{j}$, we have $t+\omega \notin \mathbb{T}$, where $\left\{t_{i j}^{k}\right\}$ is the connected points set of the timescale sequence $\left\{\mathbb{T}_{i}\right\}_{i \in \mathbb{Z}^{+}}$;

(e) $R_{0}=\{0\}$ if and only if $\mathbb{T}_{r}$ is a zero-periodic time scale and $R_{0}=\emptyset$ if and only if $\mathbb{T}_{r}=\emptyset$; and the set $\Pi$ is called a changing-periods set of $\mathbb{T}, \mathbb{T}_{i}$ is called the periodic sub-timescale of $\mathbb{T}$ and $S_{i}$ is called the periods subset of $\mathbb{T}$ or the periods set of $\mathbb{T}_{i}, \mathbb{T}_{r}$ is called the remain timescale of $\mathbb{T}$ and $R_{0}$ the remain periods set of $\mathbb{T}$.

Remark 2.4 From Definition 2.6, it follows that if $\mathbb{T}$ is a changing-periodic time scale, then the remain timescale $\mathbb{T}_{r}$ is a finite union of the closed intervals or $\mathbb{T}_{r}=\emptyset$, i.e., $R_{0}=\{0\}$ or $R_{0}=\emptyset$.

Remark 2.5 From condition (c) in Definition 2.6, for all connected points $t_{i j}^{k}, i \neq j$, we have $t_{i j}^{k} \in \mathbb{T}_{i}$ and $t_{i j}^{k} \in \mathbb{T}_{j}$, for all $\omega \in S_{i}$ and $\omega \in S_{j}$, we have $t_{i j}^{k}+\omega \in \mathbb{T}_{i} \subset \mathbb{T}$ and $t_{i j}^{k}+\omega \in \mathbb{T}_{j} \subset \mathbb{T}$.

Now we introduce the following related concept.

Definition 2.7 A changing-periodic time scale is called complete if and only if its remain timescale is an empty set, i.e, $\mathbb{T}_{r}=\emptyset$; similarly, $\mathbb{T}_{r} \neq \emptyset$ if and only if $\mathbb{T}$ is noncomplete.

We now prove the following proposition.

Proposition 2.1 All periodic time scales are particular changing-periodic time scales and complete.

Proof Let $\mathbb{T}=\mathbb{T}_{j}, S=S_{j}, j \in \mathbb{Z}^{+}$. Since $\mathbb{T}=\mathbb{T} \cup \emptyset$, by Definition 2.6 , we can take $\bigcup_{i=1}^{\infty} \mathbb{T}_{i}=\mathbb{T} \cup$ $\left(\bigcup_{i \neq j} \emptyset\right)$ and $\bigcup_{i=1}^{\infty} S_{i}=S \cup\left(\bigcup_{i \neq j} \emptyset\right)$, where $S$ is the periods set of $\mathbb{T}$, then $\mathbb{T}_{r}=\emptyset$ and $R_{0}=\emptyset$. Now by Definition 2.6 and Definition 2.7, we obtain the desired result. This completes the proof. 
Figure $1 T$ and $S$ come in pair when $T$ is periodic.

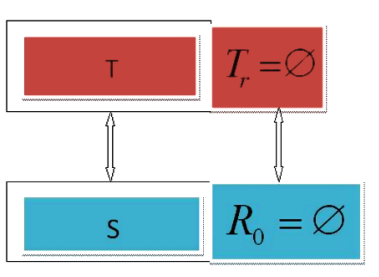

Remark 2.6 If $\mathbb{T}$ is a constant-periodic time scale, i.e., $\mathbb{T}$ is an $\omega$-periodic time scale, where $\omega$ is a constant, then $\Pi=\{n \omega: n \in \mathbb{Z}\}:=S$. We also note that although $\Pi$ can be written as $\Pi=\bigcup_{n=1}^{\infty}\{n \omega\}$, we cannot regard $S_{i}$ as $\{i \omega\}$ for all $i \in \mathbb{Z}$ since for any $i \neq j, t \in \mathbb{T}_{i}$, we have $t+j \omega \in \mathbb{T}_{i}$, which contradicts condition (d) in Definition 2.6. Therefore, $S_{i_{0}}=S, \mathbb{T}_{r}=\emptyset$ for some $i_{0} \in \mathbb{Z}^{+}$and for $i \neq i_{0}, S_{i}=\emptyset$. Hence, all constant-periodic time scales are particular changing-periodic time scales (see Figure 1).

From Definition 2.6, the following properties of changing-periodic time scales are immediate.

Theorem 2.7 Let $\mathbb{T}$ be a changing-periodic time scales, and $\mathbb{T}_{i}, S_{i}, R_{0}, \mathbb{T}_{r}$ satisfy Definition 2.6, the following hold:

(1) If $t \in \mathbb{T}$, then there must exist some $i \in \mathbb{Z}^{+}$such that $t \in \mathbb{T}_{i} \cup \mathbb{T}_{r}$. Furthermore, if $\mathbb{T}$ is complete, then $t \in \mathbb{T}_{i}$.

(2) If $t \in \mathbb{T}_{i}, \omega \in S_{i}$, then $t+\omega \in \mathbb{T}_{i} \subset \mathbb{T}$.

(3) If $i \neq j$, then $\left(\mathbb{T}_{i} \cap \mathbb{T}_{j}\right) \backslash\left\{t_{i j}^{k}\right\}=\emptyset, S_{i} \cap S_{j}=\emptyset$.

(4) If $t \in \mathbb{T}_{i} \backslash\left\{t_{i j}^{k}\right\}, t+\omega \in \mathbb{T}$ for $i \in \mathbb{Z}^{+}$, then $\omega \in S_{i}$.

(5) If $\omega \in S_{i}, t+\omega \in \mathbb{T}$ for $i \in \mathbb{Z}^{+}$, then $t \in \mathbb{T}_{i}$.

Proof From Definition 2.6, (1) and (2) are obvious, and hence we need to prove (3), (4) and (5).

To prove (3), if there exists some $t \in\left(\mathbb{T}_{i} \cap \mathbb{T}_{j}\right) \backslash\left\{t_{i j}^{k}\right\}, i \neq j$, then $t+\alpha \in \mathbb{T}_{i}, t \in \mathbb{T}_{j}, \forall \alpha \in S_{i}$ and $t+\beta \in \mathbb{T}_{j}, t \in \mathbb{T}_{i}, \forall \beta \in S_{j}$, but this contradicts condition (d) in Definition 2.6. Similarly, if there exists some $\tau \in S_{i} \cap S_{j}, i \neq j$, then $t+\tau \in \mathbb{T}_{i}, \tau \in S_{j}, \forall t \in \mathbb{T}_{i}$ and $t+\tau \in \mathbb{T}_{j}, \tau \in S_{i}$, $\forall t \in \mathbb{T}_{j}$, which also contradicts condition (d) in Definition 2.6.

To prove (4), we assume that $\omega \notin S_{i}$ : case (1) $\omega \notin R_{0}$, then there must exist $S_{j}$ such that $\omega \in$ $S_{j}, i \neq j$, but then $t+\omega \in \mathbb{T}$ for $t \in \mathbb{T}_{i} \backslash\left\{t_{i j}^{k}\right\}$, which contradicts condition (d) in Definition 2.6; case (2) $\omega \in R_{0}$, then from $t+\omega \in \mathbb{T}$, we have $t \in \mathbb{T}_{r}$, but this contradicts the fact that $t \in \mathbb{T}_{i}$.

To prove (5), we assume that $t \notin \mathbb{T}_{i}$ : case (1) $t \notin \mathbb{T}_{r}$, then there must exist $\mathbb{T}_{j}$ such that $t \in \mathbb{T}_{j}, i \neq j$ and $t$ is obviously not a connected point between $\mathbb{T}_{i}$ and $\mathbb{T}_{j}$, then $t+\omega \in \mathbb{T}$ for $\omega \in S_{i}$, which contradicts condition (d) in Definition 2.6; case (2) $t \in \mathbb{T}_{r}$, from $t+\omega \in \mathbb{T}$, we have $\omega \in R_{0}$, but this contradicts the fact that $\omega \in S_{i}$. This completes the proof.

In view of the characteristics of changing-periodic time scales (Theorem 2.7), we can introduce an index function $\tau_{t}: \mathbb{T} \rightarrow \mathbb{Z}^{+} \cup\{0\}$ such that for $t \in \mathbb{T}, t \in \mathbb{T}_{\tau_{t}}$ holds. This function plays a very important role in introducing well-defined functions on time scales. Formally, we have the following definition of $\tau_{t}$. 
Definition 2.8 Let $\mathbb{T}$ be a changing-periodic time scale, then the function $\tau$

$$
\begin{aligned}
& \tau: \mathbb{T} \mapsto \mathbb{Z}^{+} \cup\{0\}, \\
&\left(\bigcup_{i=1}^{\infty} \mathbb{T}_{i}\right) \mapsto i, \quad \text { where } t \in \mathbb{T}_{i}, i \in \mathbb{Z}^{+}, \\
& \mathbb{T}_{r} \mapsto 0, \quad \text { where } t \in \mathbb{T}_{r}, \\
& t \mapsto \tau_{t}
\end{aligned}
$$

is called an index function for $\mathbb{T}$, where the corresponding periods set of $\mathbb{T}_{\tau_{t}}$ is denoted as $S_{\tau_{t}}$. In what follows we shall call $S_{\tau_{t}}$ the adaption set generated by $t$, and all the elements in $S_{\tau_{t}}$ will be called the adaption factors for $t$.

Remark 2.8 From Definition 2.8, if $\tau_{t}$ is an index function for $\mathbb{T}$, then it immediately follows that

(i) for $t \in \mathbb{T}$, we have $t \in \mathbb{T}_{\tau_{t}}$;

(ii) for each $i \in \mathbb{Z}^{+}, t_{1}, t_{2} \in \mathbb{T}_{i}$ if and only if $\tau_{t_{1}}=\tau_{t_{2}}=i$. Furthermore, for $t_{1}, t_{2} \in \mathbb{T}_{r} \neq \emptyset$ if and only if $\tau_{t_{1}}=\tau_{t_{2}}=0$, i.e., $\mathbb{T}_{r}=\mathbb{T}_{0}$ (see Figure 2).

Obviously, if $\mathbb{T}_{r}=\emptyset$, then $\tau: \mathbb{T} \rightarrow \mathbb{Z}^{+}$.

Remark 2.9 Let $\mathbb{T}$ be a changing-periodic time scale, for all $t \in \mathbb{T}$ and all $\omega \in S_{\tau_{t}}$, we have $t+\omega \in \mathbb{T}_{\tau_{t}} \subset \mathbb{T}$. It is also obvious that if $S_{i}$ is an adaption set generated by some given $t_{0} \in \mathbb{T}_{i}$, then $S_{\tau_{t_{0}}}$ is the adaption set for all $t \in \mathbb{T}_{i}$ since $\tau_{t_{0}}=\tau_{t}=i$ for all $t \in \mathbb{T}_{i}$.

Now we can prove the following proposition.

Proposition 2.2 $\mathbb{T}$ is an $\omega$-periodic time scale if and only if we can obtain its index function $\tau_{t} \equiv z$ for all $t \in \mathbb{T}$, where $z$ denotes some positive integer.

Proof If $\mathbb{T}$ is an $\omega$-periodic time scale, then we find $S=\{n \omega: n \in \mathbb{Z}\}$, and for all $t \in \mathbb{T}$ and all $\tilde{\tau} \in S$, we have $t+\tilde{\tau} \in \mathbb{T}$. Thus, by Proposition 2.1 and Remark 2.8, there exists some

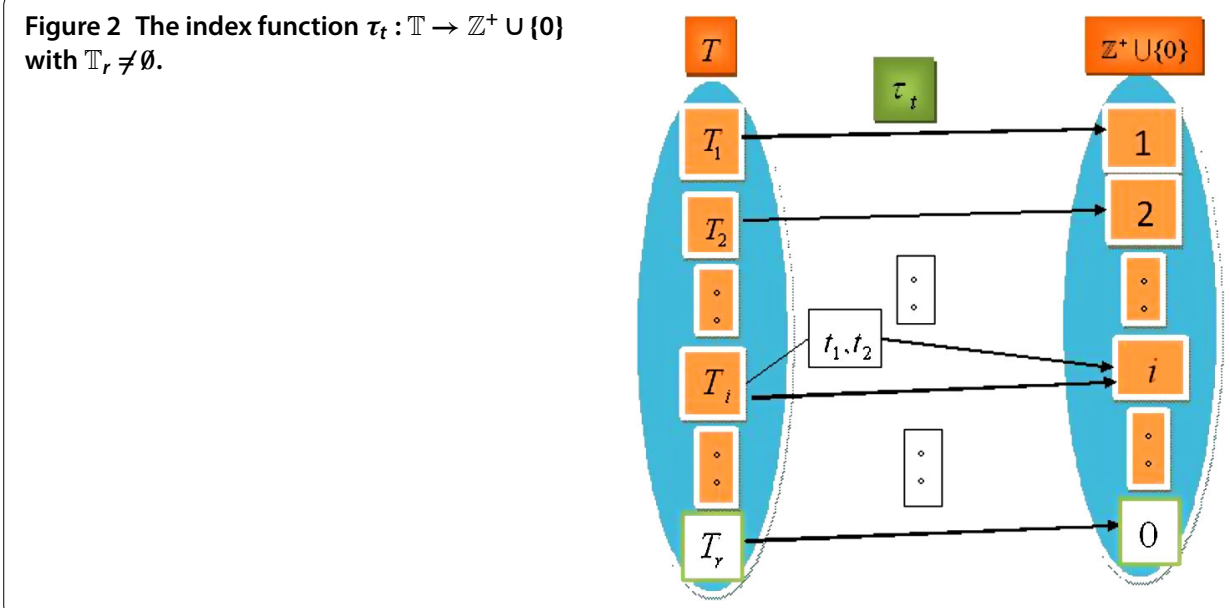


positive integer $z$ such that $\mathbb{T}=\mathbb{T}_{z}$ and $S=S_{z}$, i.e., we can choose the index function $\tau_{t} \equiv z$ for all $t \in \mathbb{T}$.

If $\tau_{t} \equiv z$ for all $t \in \mathbb{T}$, then there exists an adaption set $S_{\tau_{t}}=S_{z}$ for all $t \in \mathbb{T}$ such that for any $\omega \in S_{\tau_{t}}$, we have $t+\omega \in \mathbb{T}_{\tau_{t}}=\mathbb{T}$. Hence, $\mathbb{T}$ is $\omega$-periodic. This completes the proof.

Remark 2.10 All changing-periodic time scales can be equipped with the corresponding index functions such that for all $t \in \mathbb{T}$ and all $\omega \in S_{\tau_{t}}, t+\omega \in \mathbb{T}_{\tau_{t}} \subset \mathbb{T}$.

We are now in the position to prove the following important theorem which classifies the time scales with bounded graininess function $\mu$ as changing-periodic time scales.

Theorem 2.11 If $\mathbb{T}$ is an infinite time scale and the graininess function $\mu: \mathbb{T} \rightarrow \mathbb{R}^{+}$is bounded, then $\mathbb{T}$ is a changing-periodic time scale.

Proof Without loss of generality, we assume that $\sup \mathbb{T}=+\infty, \inf \mathbb{T}=-\infty$. We denote the set

$$
\mathbb{I}=\overline{\left\{\mathbb{T} \cap \mathbb{T}^{\tau}: \tau \in[-\bar{\mu}, \bar{\mu}] \backslash\{0\}\right\}},
$$

where $\bar{A}$ denotes the closure of the set $A$ and $\bar{\mu}=\sup _{t \in \mathbb{T}} \mu(t)$. Clearly, $\mathbb{I}$ forms a semiordered set with respect to the inclusion relation and $\mathbb{I}$ is closed. Denote $\mathbb{I}^{*}$ the any subset of $\mathbb{I}$ and is totally ordered. Hence, we can obtain two cases:

Case (1):

$$
\mathbb{I}^{*}=\left\{\left(\mathbb{T} \cap \mathbb{T}^{\tau_{n}}\right) \in \mathbb{I}: \mathbb{T}_{\tau_{n}} \supset \mathbb{T}_{\tau_{n+1}}, n \in \mathbb{N}\right\}
$$

then $\mathbb{T}_{\tau_{1}} \in \mathbb{I}^{*} \subset \mathbb{I}$ and $\mathbb{T}_{\tau_{1}}$ is an upper bound of $\mathbb{I}^{*}$ in $\mathbb{I}$.

Case (2):

$$
\mathbb{I}^{*}=\left\{\left(\mathbb{T} \cap \mathbb{T}^{\tau_{n}}\right) \in \mathbb{I}: \mathbb{T}_{\tau_{n}} \subset \mathbb{T}_{\tau_{n+1}}, n \in \mathbb{N}\right\}
$$

then $\lim _{n \rightarrow \infty}\left(\mathbb{T} \cap \mathbb{T}^{\tau_{n}}\right)=\bigcup_{n=1}^{\infty}\left(\mathbb{T} \cap \mathbb{T}^{\tau_{n}}\right)=\mathbb{T} \cap \mathbb{T}^{\tau_{\infty}} \subset \mathbb{T}$ is an upper bound of $\mathbb{I}^{*}$. Because $\mathbb{I}$ is closed, then $\mathbb{T} \cap \mathbb{T}^{\tau_{\infty}} \in \mathbb{I}$. According to Zorn's lemma (i.e., Lemma 2.1), there exists some $\tau_{0} \in[-\bar{\mu}, \bar{\mu}] \backslash\{0\}$ such that $\mathbb{T} \cap \mathbb{T}^{\tau_{0}}$ is the maximum element in $\mathbb{I}$. Note that since $\mu$ is bounded, $\mathbb{T} \cap \mathbb{T}^{\tau_{0}} \neq \emptyset$ and $\sup \left(\mathbb{T} \cap \mathbb{T}^{\tau_{0}}\right)=+\infty, \inf \left(\mathbb{T} \cap \mathbb{T}^{\tau_{0}}\right)=-\infty$.

Now we will show that $\mathbb{T}$ is a changing-periodic time scale. We divide the proof into the following steps.

Step I. We can find a time scale $\mathbb{T}_{0}^{1}$ such that $\mathbb{T}_{0}^{1} \subset \mathbb{T}$ is the largest periodic sub-timescale in $\mathbb{T}$. For this, we make a continuous translation of $\mathbb{T}$ to find a number $\tau_{1}$ such that $\mathbb{T} \cap \mathbb{T}^{\tau_{1}}:=$ $\mathbb{T}_{1}$ is the maximum. Next, consider a translation of $\mathbb{T}_{1}$ again to find a number $\tau_{2}$ such that $\mathbb{T}_{1} \cap \mathbb{T}_{1}^{\tau_{2}}:=\mathbb{T}_{2}$ is the maximum. Continue this process $n$ times to find a number $\tau_{n}$ such that $\mathbb{T}_{n-1} \cap \mathbb{T}_{n-1}^{\tau_{n}}:=\mathbb{T}_{n}$ is the maximum. This process leads to a decreasing sequence of timescale sets:

$$
\mathbb{T} \supset \mathbb{T}_{1} \supset \mathbb{T}_{2} \supset \cdots \supset \mathbb{T}_{n} \supset \cdots
$$


Hence, it follows that $\lim _{n \rightarrow \infty} \mathbb{T}_{n}=\mathbb{T}_{0}^{1}$. This shows that there exists some $\tau_{0}^{1}$ such that $\mathbb{T}_{0}^{1}$ coincides with itself through the translation of this number. Obviously, this also implies that $\mathbb{T}_{0}^{1}$ is not a finite union of the closed intervals.

Now we claim that $\mathbb{T}_{0}^{1} \neq \emptyset$. In fact, if $\mathbb{T}_{0}^{1}=\emptyset$, then there exists some sufficiently large $n_{0}$ such that $\mathbb{T}_{n_{0}}=\emptyset$, i.e., there exists some sub-timescale $\mathbb{T}_{n_{0}-1} \subset \mathbb{T}$ such that $\mathbb{T}_{n_{0}-1}$ has no intersection with itself through the translation of the number $\tau_{n_{0}}$ and $\mathbb{T}_{n_{0}-1} \cap \mathbb{T}_{n_{0}-1}^{\tau_{n_{0}}}$ is the maximum element in

$$
\mathbb{I}_{n_{0}}=\left\{\mathbb{T}_{n_{0}-1} \cap \mathbb{T}_{n_{0}-1}^{\tau}: \tau \neq 0\right\}=\emptyset,
$$

which means that $\mathbb{T}_{n_{0}-1}$ is a single point set, but this is a contradiction since sup $\mathbb{T}_{n_{0}-1}=$ $+\infty$, inf $\mathbb{T}_{n_{0}-1}=-\infty$. Therefore, $\mathbb{T}_{0}^{1}$ is a $\tau_{0}^{1}$-periodic sub-timescale.

Step II. For the time scale $\mathbb{T}_{1}^{*}:=\overline{\mathbb{T} \backslash \mathbb{T}_{0}^{1}}$, where $\bar{A}$ denotes the closure of the set $A$, by replacing $\mathbb{T}$ with $\mathbb{T}_{1}^{*}$ and repeating Step $\mathrm{I}$, we can obtain the periodic sub-timescale $\mathbb{T}_{0}^{2}$. For the time scale $\mathbb{T}_{2}^{*}:=\overline{\mathbb{T} \backslash\left(\mathbb{T}_{0}^{1} \cup \mathbb{T}_{0}^{2}\right)}$, by replacing $\mathbb{T}$ with $\mathbb{T}_{2}^{*}$ and repeating Step I, we can obtain the periodic sub-timescale $\mathbb{T}_{0}^{3}$. Similarly, we can obtain $\mathbb{T}_{0}^{4}, \ldots, \mathbb{T}_{0}^{n} \ldots$ Obviously, the timescale sequence $\left\{\mathbb{T}_{0}^{i}\right\}_{i \in \mathbb{Z}^{+}}$is well connected and $\left(\mathbb{T}_{0}^{i} \cap \mathbb{T}_{0}^{j}\right) \backslash\left\{t_{i j 0}^{k}\right\}=\emptyset$ for $i \neq j$, where $\left\{t_{i j 0}^{k}\right\}$ is the connected points set between $\mathbb{T}_{0}^{i}$ and $\mathbb{T}_{0}^{j}$. If for some sufficiently large $n_{0}$, still $\mathbb{T}_{n_{0}}^{*}=\overline{\mathbb{T} \backslash \bigcup_{i=1}^{n_{0}} \mathbb{T}_{0}^{i}}$ is an infinite time scale, then we repeat Step I again until the remaining timescale $\mathbb{T} \backslash \bigcup_{i=1}^{\infty} \mathbb{T}_{0}^{i}=\emptyset$, or a finite union of the closed intervals.

Step III. Letting the set $\Pi$ of $\mathbb{T}$ be as

$$
\Pi=\left(\bigcup_{i=1}^{\infty} S_{i}\right) \cup R_{0}=\left(\bigcup_{i=1}^{\infty}\left\{n \tau_{0}^{i}, n \in \mathbb{Z}\right\}\right) \cup R_{0},
$$

where $R_{0}=\{0\}$ or $\emptyset$, from Steps I and II, it follows that $S_{i} \cap S_{j}=\emptyset$ if $i \neq j$.

From Steps I, II, III, we find $\left(\bigcup_{i=1}^{\infty} \mathbb{T}_{0}^{i}\right) \cup\left(\bigcup_{i=1}^{k}\left[\alpha_{i}, \beta_{i}\right]\right)=\mathbb{T}$, where $k$ is some finite number and $\left[\alpha_{i}, \beta_{i}\right]$ are closed intervals for $i=1,2, \ldots, k$, or $\left(\bigcup_{i=1}^{\infty} \mathbb{T}_{0}^{i}\right)=\mathbb{T}$. Therefore, $\mathbb{T}$ is a changing-periodic time scale. This completes the proof.

From the proof of Theorem 2.11, we have the following proposition.

Proposition 2.3 If $\mathbb{T}=\bigcup_{i=1}^{\infty} \mathbb{T}_{i}$, where $\mathbb{T}_{i}$ is $\omega_{i}$-periodic for each $i \in \mathbb{Z}^{+}$, then there exists a well-connected timescale sequence $\left\{\mathbb{T}_{0}^{i}\right\}_{i \in \mathbb{Z}^{+}}$such that $\mathbb{T}=\bigcup_{i=1}^{\infty} \mathbb{T}_{0}^{i}$, where $\mathbb{T}_{0}^{i}$ is $\omega_{0}^{i}$-periodic. Furthermore, $\mathbb{T}$ is a complete changing-periodic time scale.

Proof Since $\mathbb{T}_{i}$ is periodic for each $i \in \mathbb{Z}^{+}$, the graininess function $\mu_{i}: \mathbb{T}_{i} \rightarrow \mathbb{R}^{+}$is bounded for each $i \in \mathbb{Z}^{+}$. Thus, the graininess function $\mu$ of $\mathbb{T}$ is also bounded. Therefore, in view of Theorem 2.11, $\mathbb{T}$ is a changing-periodic time scale. Further, from the proof of Theorem 2.11, $\mathbb{T}$ can be decomposed into the union of all elements in the well-connected periodic timescale sequence $\left\{\mathbb{T}_{0}^{i}\right\}_{i \in \mathbb{Z}^{+}}$and $\mathbb{T}_{r}=\emptyset$. Now, by Definition 2.6 and Definition 2.7, $\mathbb{T}$ is a complete changing-periodic time scale. This completes the proof.

Now we shall demonstrate some complete changing-periodic time scales.

Example 2.12 Let $k \in \mathbb{Z}$, consider the following time scale:

$$
\mathbb{T}=\left\{\bigcup_{k \in(-\infty,+\infty) \backslash\{0\}}\left[\frac{9}{7} k, \frac{9}{7} k+\frac{1}{17}\right]\right\} \cup\left\{\bigcup_{k \in(-\infty,+\infty) \backslash\{0\}}\left[\frac{5}{3} k, \frac{5}{3} k+\frac{1}{15}\right]\right\} .
$$


We denote

$$
\mathbb{T}_{1}=\bigcup_{k \in(-\infty,+\infty) \backslash\{0\}}\left[\frac{9}{7} k, \frac{9}{7} k+\frac{1}{17}\right] \text { and } \mathbb{T}_{2}=\bigcup_{k \in(-\infty,+\infty) \backslash\{0\}}\left[\frac{5}{3} k, \frac{5}{3} k+\frac{1}{15}\right]
$$

Then, by a direct calculation, the set $\Pi$ is

$$
\Pi=\left\{\frac{9}{7} n, n \in \mathbb{Z}\right\} \cup\left\{\frac{5}{3} n, n \in \mathbb{Z}\right\}:=S_{1} \cup S_{2} .
$$

This time scale is a changing-periodic time scale according to Definition 2.6.

Example 2.13 Let $k \in \mathbb{Z}$, consider the following time scale:

$$
\begin{aligned}
\mathbb{T}= & \left\{\bigcup_{k \in(-\infty,+\infty) \backslash\{0\}}\left[\frac{79}{19} k, \frac{79}{19} k+\frac{2}{190}\right]\right\} \cup\left\{\bigcup_{k \in(-\infty,+\infty) \backslash\{0\}}\left[\frac{71}{10} k, \frac{71}{10} k+\frac{2}{170}\right]\right\} \\
& \cup\left\{\bigcup_{k \in(-\infty,+\infty) \backslash\{0\}}\left[\frac{86}{17} k, \frac{86}{17} k+\frac{3}{170}\right]\right\} .
\end{aligned}
$$

We denote

$$
\begin{aligned}
& \mathbb{T}_{1}=\bigcup_{k \in(-\infty,+\infty) \backslash\{0\}}\left[\frac{79}{19} k, \frac{79}{19} k+\frac{2}{190}\right], \quad \mathbb{T}_{2}=\bigcup_{k \in(-\infty,+\infty) \backslash\{0\}}\left[\frac{71}{10} k, \frac{71}{10} k+\frac{2}{170}\right], \\
& \mathbb{T}_{3}=\bigcup_{k \in(-\infty,+\infty) \backslash\{0\}}\left[\frac{86}{17} k, \frac{86}{17} k+\frac{3}{170}\right] .
\end{aligned}
$$

Then, by a direct calculation, the set $\Pi$ is

$$
\Pi=\left\{\frac{79}{19} n, n \in \mathbb{Z}\right\} \cup\left\{\frac{71}{10} n, n \in \mathbb{Z}\right\} \cup\left\{\frac{86}{17} n, n \in \mathbb{Z}\right\}:=S_{1} \cup S_{2} \cup S_{3} .
$$

This time scale is a changing-periodic time scale according to Definition 2.6.

Example 2.14 Let $k \in \mathbb{Z}$, consider the following time scale:

$$
\mathbb{T}=\left\{\bigcup_{k \in(-\infty,+\infty) \backslash\{0\}}\left[\frac{3}{2} k, \frac{3}{2} k+\frac{1}{12}\right]\right\} \cup\left\{\bigcup_{k \in(-\infty,+\infty) \backslash\{0\}}\left[\frac{3 \sqrt{2}}{2} k, \frac{3 \sqrt{2}}{2} k+\frac{\sqrt{3}}{5}\right]\right\} .
$$

We denote

$$
\mathbb{T}_{1}=\bigcup_{k \in(-\infty,+\infty) \backslash\{0\}}\left[\frac{3}{2} k, \frac{3}{2} k+\frac{1}{12}\right] \text { and } \mathbb{T}_{2}=\bigcup_{k \in(-\infty,+\infty) \backslash\{0\}}\left[\frac{3 \sqrt{2}}{2} k, \frac{3 \sqrt{2}}{2} k+\frac{\sqrt{3}}{5}\right]
$$

Then, by a direct calculation, the set $\Pi$ is

$$
\Pi=\left\{\frac{3}{2} n, n \in \mathbb{Z}\right\} \cup\left\{\frac{3 \sqrt{2}}{2} n, n \in \mathbb{Z}\right\}:=S_{1} \cup S_{2} .
$$

This time scale is a changing-periodic time scale according to Definition 2.6. 
The above examples lead to the following immediate propositions.

Proposition 2.4 Let $\mathbb{T}_{i}$ be constant-periodic time scales for all $i \in I$, then $\bigcup_{i \in I} \mathbb{T}_{i}$ may not be a constant-periodic time scale, where I is an index number set.

Proposition 2.5 Let $\mathbb{T}_{i}$ be constant-periodic time scales with $\omega_{i}$-period for all $i \in I$, and $\omega_{i}$ is a natural number for each $i \in I$. If all the numbers in the set $\left\{\omega_{i}\right\}_{i \in I}$ have a lowest common multiple $\omega$, then $\bigcup_{i \in I} \mathbb{T}_{i}$ is an $\omega$-periodic time scale.

Example 2.15 Let $k \in \mathbb{Z}$, consider the following time scale:

$$
\begin{aligned}
\mathbb{T}= & \left\{\bigcup_{k \in(-\infty,+\infty) \backslash\{0\}}\left[2 k, 2 k+\frac{1}{23}\right]\right\} \cup\left\{\bigcup_{k \in(-\infty,+\infty) \backslash\{0\}}\left[3 k, 3 k+\frac{1}{16}\right]\right\} \\
& \cup\left\{\bigcup_{k \in(-\infty,+\infty) \backslash\{0\}}\left[5 k, 5 k+\frac{1}{37}\right]\right\},
\end{aligned}
$$

then it is easily seen that $\Pi=\{2 n, n \in \mathbb{Z}\} \cup\{3 n, n \in \mathbb{Z}\} \cup\{5 n, n \in \mathbb{Z}\}$, and $\mathbb{T}$ has the constant period 30.

Now we construct some changing-periodic time scales with $\mu$ bounded.

Example 2.16 Let $a>1, t>a, t \in \mathbb{T}$, and consider the following time scale:

$$
\mathbb{P}_{a, e^{-t}}=\bigcup_{m=1}^{\infty}\left[p_{m}, a+p_{m}\right],
$$

where

$$
\begin{aligned}
p_{m}= & (m-1) a+\sum_{k=1}^{m-1} \exp \{-[k a+\exp (-a)+\exp (-(2 a+\exp (-a)))+\cdots \\
& \underbrace{+\exp (-((k-1) a+\exp (-a)))]}_{k \text { terms }}\} .
\end{aligned}
$$

Then we have

$$
\sigma(t)= \begin{cases}t, & \text { if } t \in \bigcup_{m=1}^{\infty}\left[p_{m}, a+p_{m}\right), \\ t+e^{-t}, & \text { if } t \in \bigcup_{m=1}^{\infty}\left\{a+p_{m}\right\}\end{cases}
$$

and

$$
\mu(t)= \begin{cases}0, & \text { if } t \in \bigcup_{m=1}^{\infty}\left[p_{m}, a+p_{m}\right), \\ e^{-t}, & \text { if } t \in \bigcup_{m=1}^{\infty}\left\{a+p_{m}\right\} .\end{cases}
$$

Similarly, we also have

$$
\rho(t)= \begin{cases}t, & \text { if } t \in \bigcup_{m=1}^{\infty}\left(p_{m}, a+p_{m}\right], \\ t-e^{-t}, & \text { if } t \in \bigcup_{m=1}^{\infty}\left\{p_{m}\right\}\end{cases}
$$


and

$$
v(t)= \begin{cases}0, & \text { if } t \in \bigcup_{m=1}^{\infty}\left(p_{m}, a+p_{m}\right] \\ e^{-t}, & \text { if } t \in \bigcup_{m=1}^{\infty}\left\{p_{m}\right\}\end{cases}
$$

Example 2.17 Let $a>1$ and consider the following almost periodic time scale:

$$
\mathbb{P}_{a,|\sin \sqrt{3} t+\sin \sqrt{7} t|}=\bigcup_{m=1}^{\infty}\left[p_{m}, a+p_{m}\right]
$$

where

$$
\begin{aligned}
& p_{m}=(m-1) a+\sum_{k=1}^{m-1} \mid \sin \sqrt{3}(k a+|\sin \sqrt{3} a+\sin \sqrt{7} a| \\
&+\mid \sin \sqrt{3}(2 a+|\sin \sqrt{3} a+\sin \sqrt{7} a|) \\
&+\sin \sqrt{7}(2 a+|\sin \sqrt{3} a+\sin \sqrt{7} a|) \mid \\
&+\cdots+\mid \sin \sqrt{3}((k-1) a+|\sin \sqrt{3} a+\sin \sqrt{7} a|) \\
&+\sin \sqrt{7}((k-1) a+|\sin \sqrt{3} a+\sin \sqrt{7} a|) \mid) \\
&+\sin \sqrt{7}(k a+|\sin \sqrt{3} a+\sin \sqrt{7} a| \\
&+\mid \sin \sqrt{3}(2 a+|\sin \sqrt{3} a+\sin \sqrt{7} a|) \\
&+\sin \sqrt{7}(2 a+|\sin \sqrt{3} a+\sin \sqrt{7} a|) \mid \\
&+\cdots+\mid \sin \sqrt{3}((k-1) a+|\sin \sqrt{3} a+\sin \sqrt{7} a|) \\
&+\sin \sqrt{7}((k-1) a+|\sin \sqrt{3} a+\sin \sqrt{7} a|) \mid) \mid . \\
& k \text { terms }
\end{aligned}
$$

Then we have

$$
\sigma(t)= \begin{cases}t, & \text { if } t \in \bigcup_{m=1}^{\infty}\left[p_{m}, a+p_{m}\right), \\ t+|\sin \sqrt{3} t+\sin \sqrt{7} t|, & \text { if } t \in \bigcup_{m=1}^{\infty}\left\{a+p_{m}\right\}\end{cases}
$$

and

$$
\mu(t)= \begin{cases}0, & \text { if } t \in \cup_{m=1}^{\infty}\left[p_{m}, a+p_{m}\right), \\ |\sin \sqrt{3} t+\sin \sqrt{7} t|, & \text { if } t \in \cup_{m=1}^{\infty}\left\{a+p_{m}\right\}\end{cases}
$$

Remark 2.18 In [14] it has been shown that time scales considered in Examples 2.16 and 2.17 are almost periodic time scales. In fact, in our next corollary we shall show that all concepts of almost periodic time scales discussed in $[13,14]$ are actually changing-periodic time scales.

Corollary 2.19 Almost periodic time scales $(c f .[13,14])$ are particular changing-periodic time scales. 


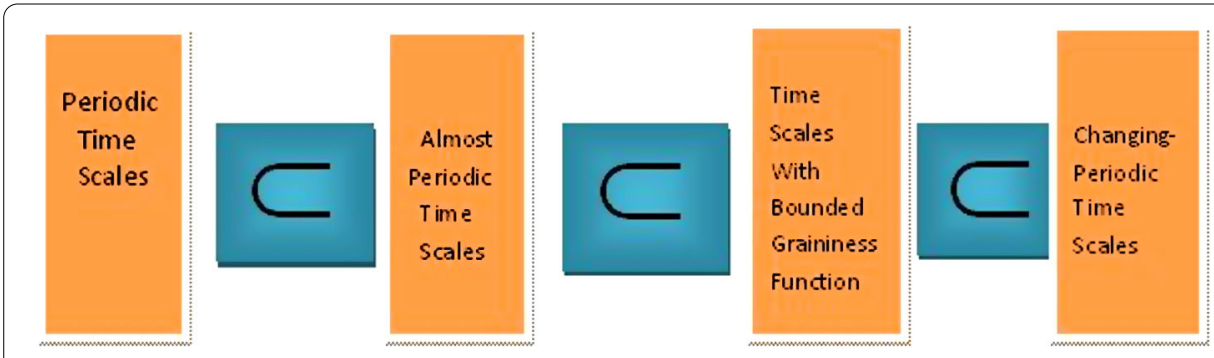

Figure 3 The containing relationship of four classes of time scales.

Proof Obviously, if $\mathbb{T}$ is an almost periodic time scale, then $\mu: \mathbb{T} \rightarrow \mathbb{R}^{+}$is bounded, and thus, by Theorem $2.11, \mathbb{T}$ is a changing-periodic time scale. This completes the proof.

Remark 2.20 Time scales considered in Examples 2.12, 2.13 and 2.14 are not almost periodic time scales but changing-periodic time scales. Therefore, changing-periodic time scales strictly include almost periodic time scales (see Figure 3 ).

Now we state and prove the following theorem, which plays an important role in establishing classical functions on changing-periodic time scales.

Theorem 2.21 (Decomposition theorem of time scales) Let $\mathbb{T}$ be an infinite time scale and the graininess function $\mu: \mathbb{T} \rightarrow \mathbb{R}^{+}$be bounded, then $\mathbb{T}$ is a changing-periodic time scale, i.e., there exists a countable periodic decomposition such that $\mathbb{T}=\left(\bigcup_{i=1}^{\infty} \mathbb{T}_{i}\right) \cup \mathbb{T}_{r}$ and $\mathbb{T}_{i}$ is an $\omega$-periodic sub-timescale, $\omega \in S_{i}, i \in \mathbb{Z}^{+}$, where $\mathbb{T}_{i}, S_{i}, \mathbb{T}_{r}$ satisfy the conditions in Definition 2.6.

Proof From Theorem 2.11, we know that $\mathbb{T}$ is a changing-periodic time scale, so one can obtain the decomposition of the time scale $\mathbb{T}$ directly from Definition 2.6. The proof is complete.

Remark 2.22 From the definition of the index function (i.e., Definition 2.8), we see that a decomposition of a time scale can be determined by its index function $\tau$. In fact, as a consequence of Theorem 2.21, we have the following result.

Theorem 2.23 (Periodic coverage theorem of time scales) Let $\mathbb{T}$ be an infinite time scale and the graininess function $\mu: \mathbb{T} \rightarrow \mathbb{R}^{+}$be bounded, then $\mathbb{T}$ can be covered by countable periodic time scales.

Proof From Theorem 2.21 and Definition 2.6, it follows that

$$
\mathbb{T}=\left(\bigcup_{i=1}^{\infty} \mathbb{T}_{i}\right) \cup \mathbb{T}_{r},
$$

where $\mathbb{T}_{i}$ is periodic for each $i \in \mathbb{Z}^{+}$and $\mathbb{T}_{r}=\emptyset$ or $\mathbb{T}_{r}$ is a zero-periodic time scale. This completes the proof.

It is interesting to note that in view of Theorem 2.21, Proposition 2.3 and Definition 2.6, it is possible to introduce another concept of changing-periodic time scales. 


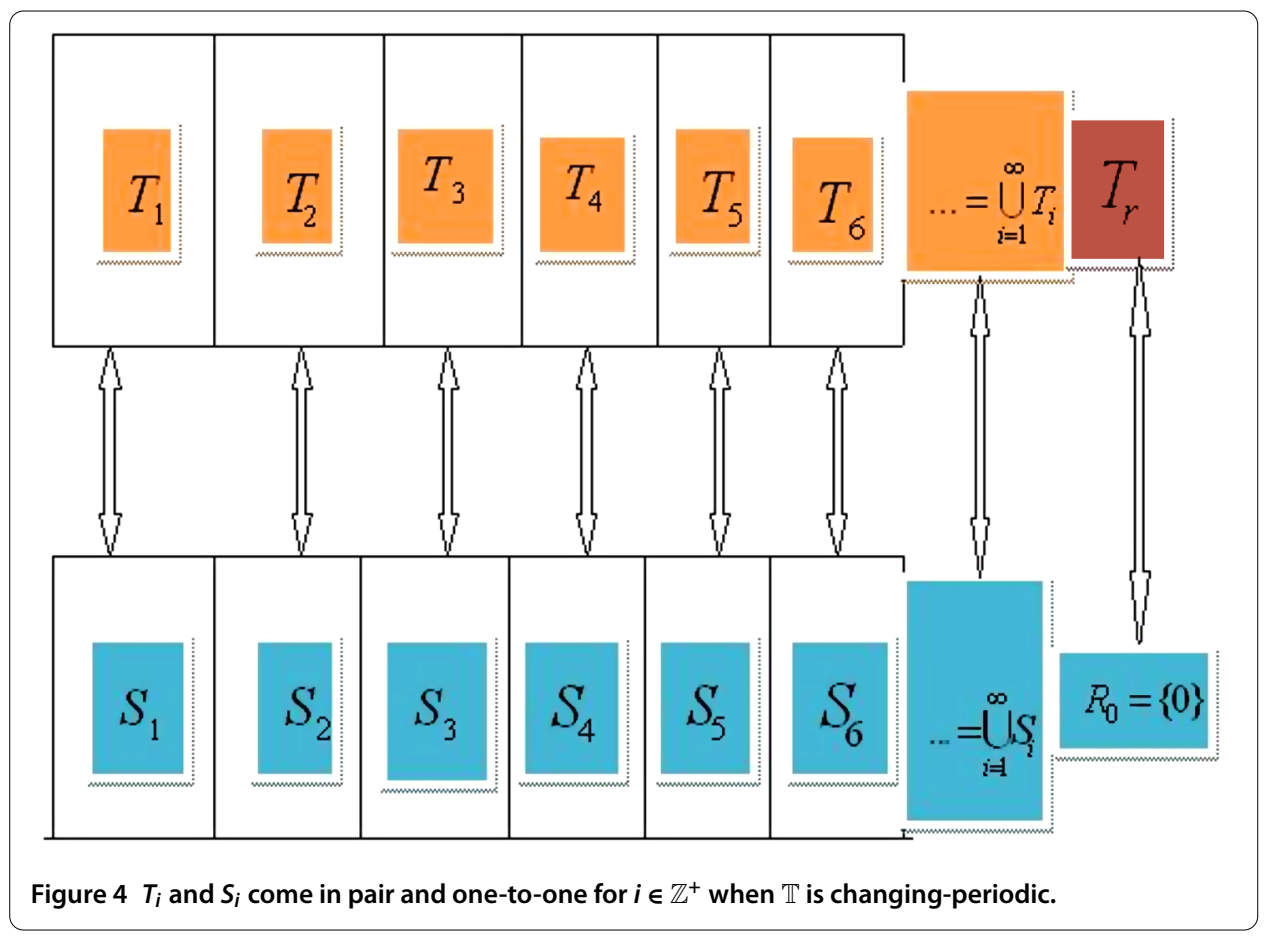

Definition 2.9 We say that $\mathbb{T}$ is a changing-periodic time scale if and only if $\mathbb{T}$ is a countable union of periodic time scales.

Remark 2.24 According to Remark 2.2, a finite union of closed intervals $\bigcup_{i=1}^{k}\left[\alpha_{i}, \beta_{i}\right]:=\mathbb{T}_{r}$ is a zero-periodic time scale. Thus, $\mathbb{T}=\left(\bigcup_{i=1}^{\infty} \mathbb{T}_{i}\right) \cup \mathbb{T}_{r}$ can be regarded as a union of periodic time scales. Therefore, by Proposition 2.3, Definition 2.6 is equivalent to Definition 2.9.

Remark 2.25 From the above Definitions 2.6 and 2.9, we note that $\mathbb{T}_{i}$ and $S_{i}$ appear in pairs for $i \in \mathbb{Z}^{+}$, likewise, $\mathbb{T}_{r}$ and $R_{0}$ also crop up in pair (see Figure 4 ).

Remark 2.26 For simplicity, since the remain timescale $\mathbb{T}_{r}$ can be regarded as the zeroperiodic time scale, a changing-periodic time scale can be denoted as $\mathbb{T}=\bigcup_{i=1}^{\infty} \mathbb{T}_{i}$ which contains $\mathbb{T}_{r}$.

\section{Almost periodicity and almost automorphy of functions on changing-periodic time scales}

By virtue of Section 2, we now propose a completely new concept of almost periodic functions on changing-periodic time scales, which includes not only the concept of almost periodic functions on periodic time scales, but also the concept of almost periodic functions on almost periodic time scales, and it is more general and comprehensive. For this, we need the following notations: Let $\alpha^{\tau}=\left\{\alpha_{n}^{\tau}\right\} \subset S_{\tau_{t}}$ and $\beta^{\tau}=\left\{\beta_{n}^{\tau}\right\} \subset S_{\tau_{t}}$ be two adaption factors sequences for $t$ under the index function $\tau$. Then $\beta^{\tau} \subset \alpha^{\tau}$ means that $\beta^{\tau}$ is a subsequence of $\alpha^{\tau} ; \alpha^{\tau}+\beta^{\tau}=\left\{\alpha_{n}^{\tau}+\beta_{n}^{\tau}\right\} ;-\alpha^{\tau}=\left\{-\alpha_{n}^{\tau}\right\} ; \mathbb{E}^{n}$ denotes $\mathbb{R}^{n}$ or $\mathbb{C}^{n}, D$ denotes an open set in $\mathbb{E}^{n}$ or $D=\mathbb{E}^{n}$, and $S$ denotes an arbitrary compact subset of $D$. We will also need the translation operator $T_{\alpha^{\tau}}, T_{\alpha^{\tau}} f(t, x)=g(t, x)$, which means that $g(t, x)=\lim _{n \rightarrow+\infty} f\left(t+\alpha_{n}^{\tau}, x\right)$ provided the limit exists. 
Definition 3.1 Let $\mathbb{T}$ be a changing-periodic time scale, i.e., $\mathbb{T}$ satisfies Definition 2.6. A function $f \in C\left(\mathbb{T} \times D, \mathbb{E}^{n}\right)$ is called a local-almost periodic function in $t \in \mathbb{T}$ uniformly for $x \in D$ if the $\varepsilon$-translation numbers set of $f$,

$$
E\{\varepsilon, f, S\}=\left\{\tilde{\tau} \in S_{\tau_{t}}:|f(t+\tilde{\tau}, x)-f(t, x)|<\varepsilon \text { for all }(t, x) \in \mathbb{T} \times S\right\}
$$

is a relatively dense set for all $\varepsilon>0$ and for each compact subset $S$ of $D$; that is, for any given $\varepsilon>0$ and each compact subset $S$ of $D$, there exists a constant $l(\varepsilon, S)>0$ such that each interval of length $l(\varepsilon, S)$ contains $\tilde{\tau}(\varepsilon, S) \in E\{\varepsilon, f, S\}$ such that

$$
|f(t+\tilde{\tau}, x)-f(t, x)|<\varepsilon \quad \text { for all }(t, x) \in \mathbb{T} \times S ;
$$

here, $\tilde{\tau}$ is called the $\varepsilon$-local translation number of $f$ and $l(\varepsilon, S)$ is called the local inclusion length of $E\{\varepsilon, f, S\}$.

Remark 3.1 Since the changing-periodic time scales include periodic and almost periodic time scales, if $\mathbb{T}$ is a $\tilde{\tau}$-periodic time scale, then $\mathbb{T}_{r}=\emptyset, R_{0}=\emptyset$ and $S_{\tau_{t}}=\{n \tilde{\tau}: n \in \mathbb{Z}\}$, so Definition 3.1 is equivalent to Definition 3.10 in [13]; if $\mathbb{T}$ is an almost periodic time scale, then $\mu$ is bounded, so $\mathbb{T}$ is a changing-periodic time scale, then Definition 3.1 includes Definition 14 in [14] since Definition 3.1 covers the almost periodicity on the part $\mathbb{T} \backslash(\mathbb{T} \cap$ $\mathbb{T}^{-\tau}$ ) of Definition 14 in [14].

Remark 3.2 In Definition 3.1, we require $E\{\varepsilon, f, S\}$ to be a relatively dense set. Thus, by the definition and the property of $S_{\tau_{t}}$, we can obtain the local almost periodicity on the periodic sub-timescale $\mathbb{T}_{\tau_{t}}$ from Definition 3.1 (see Figure 5).

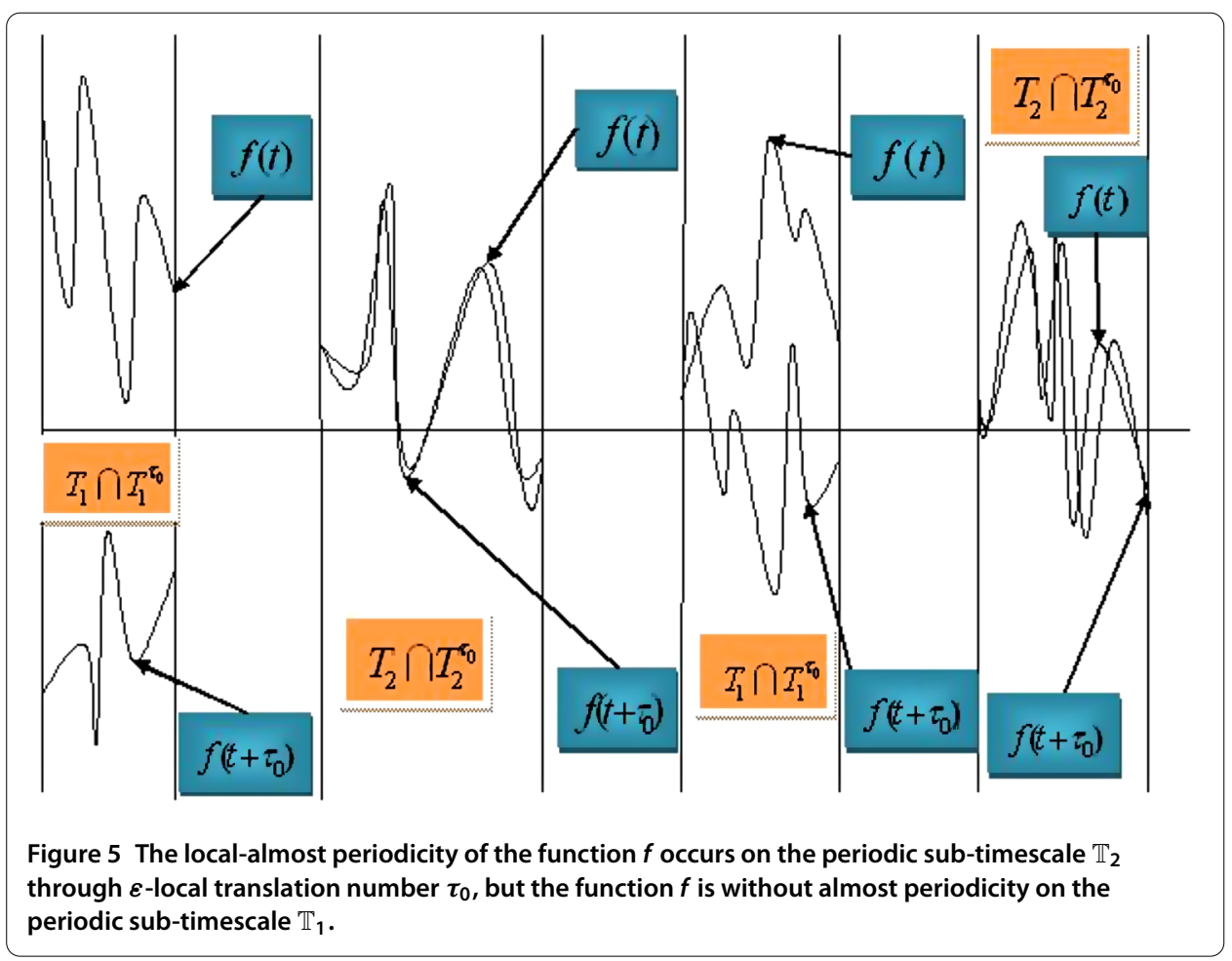


Now we give another definition which in view of Theorem 2.21 is equivalent to Definition 3.1.

Definition 3.2 Assume that $\mathbb{T}$ is a changing-periodic time scale. Let $f(t, x) \in C\left(\mathbb{T} \times D, \mathbb{E}^{n}\right)$ if for any given adaption factors sequence $\left(\alpha^{\tau}\right)^{\prime} \subset S_{\tau_{t}}$, there exists a subsequence $\alpha^{\tau} \subset\left(\alpha^{\tau}\right)^{\prime}$ such that $T_{\alpha^{\tau}} f(t, x)$ exists uniformly on $\mathbb{T} \times S$, then $f(t, x)$ is called a local-almost periodic function in $t$ uniformly for $x \in D$.

Example 3.3 Consider the changing-periodic time scale given in Example 2.17, with $a=3$, and define

$$
f(t)= \begin{cases}\sin \sqrt{7} t+\frac{1}{7} \sin t+\cos \sqrt{5} t, & \text { if } t \in \bigcup_{m=1}^{\infty}\left[p_{m}, a+p_{m}\right), \\ e^{-2 t} \cos t+5 e^{\frac{t}{100}} \sin \sqrt{13} t, & \text { if } t \in \bigcup_{m=1}^{\infty}\left\{a+p_{m}\right\}\end{cases}
$$

then $f(t)$ is a local-almost periodic function on $\mathbb{T}$. It is worth noting that $f(t)$ is almost periodic only on a local part of this time scale. In fact, if $t \in \bigcup_{m=1}^{\infty}\left\{a+p_{m}\right\}$, then the function $f(t)$ is not almost periodic on these points since $f(t)$ becomes unbounded as $t$ increases. Hence, $f(t)$ is only a local-almost periodic function on the subset of $\bigcup_{m=1}^{\infty}\left[p_{m}, a+p_{m}\right)$. From Figure 6, and in view of Theorem 2.21, it is clear that $f(t)$ is local-almost periodic on the periodic sub-timescale of the set $\bigcup_{m=1}^{\infty}\left[p_{m}, a+p_{m}\right)$, except at the right-scattered points $\bigcup_{m=1}^{\infty}\left\{a+p_{m}\right\}$, and thus by the definition of $f(t)$, it will not be almost periodic.

From Definition 3.2, we have the following proposition.

Proposition 3.1 Let $\mathbb{T}$ be a changing-periodic time scale. If $f \in C\left(\mathbb{T} \times D, \mathbb{E}^{n}\right)$ is a localalmost periodic in $t$ uniformly for $x \in D$, then $f \in C\left(\mathbb{T}_{0} \times D, \mathbb{E}^{n}\right)$ is local-almost periodic in $t$ uniformly for $x \in D$, where $\mathbb{T}_{0}$ is a changing-periodic time scale and $\mathbb{T}_{0} \subset \mathbb{T}$.

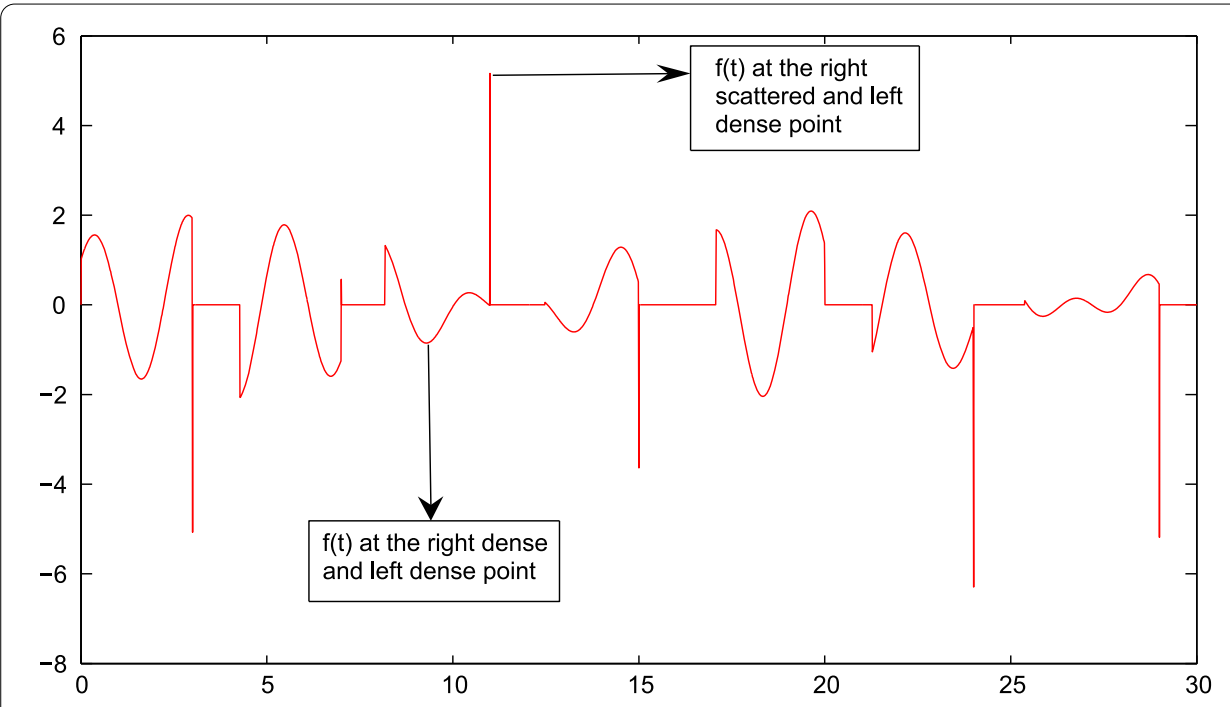

Figure $6 f(t)$ is a local-almost periodic function on the periodic sub-timescale of the set $\bigcup_{m=1}^{\infty}\left[p_{m}, a+p_{m}\right)$, except at the right-scattered points $\bigcup_{m=1}^{\infty}\left\{a+p_{m}\right\}, f(t)$ will lose its almost periodicity. 
Proof Let $f \in C\left(\mathbb{T} \times D, \mathbb{E}^{n}\right)$ be uniformly local-almost periodic, then, by Definition 3.2, for any adaption factors sequence $\left(\alpha^{\tau}\right)^{\prime} \subset S_{\tau_{t}} \subset \Pi$, there exists a subsequence $\alpha^{\tau} \subset\left(\alpha^{\tau}\right)^{\prime}$ such that $T_{\alpha^{\tau}} f(t, x)$ exists uniformly on $\mathbb{T} \times S$, where $S$ is any compact set in $D$. Consequently, $T_{\alpha^{\tau}} f(t, x)$ exists uniformly on $\mathbb{T}_{0} \times S$. This completes the proof.

Next, we have the following definition.

Definition 3.3 Let $f, g \in C\left(\mathbb{T} \times D, \mathbb{E}^{n}\right)$ be uniformly local-almost periodic and $\mathbb{T}$ be a changing-periodic time scale. We say $f$ and $g$ are synchronously local-almost periodic if $f, g$ are almost periodic on the same periodic sub-timescales of $\mathbb{T}$.

From Theorem 2.21, we can deduce the following result for synchronously local-almost periodic functions.

Theorem 3.4 If $f, g \in C\left(\mathbb{T} \times D, \mathbb{E}^{n}\right)$ are two synchronously local-almost periodic functions, then, for any $\varepsilon>0$, the intersection of $\varepsilon$-local translation numbers sets of $f$ and $g$ is a nonempty relatively dense set, i.e., $E\{\varepsilon, f, S\} \cap E\{\varepsilon, g, S\}$ is a nonempty relatively dense set.

Proof If $f, g \in C\left(\mathbb{T} \times D, \mathbb{E}^{n}\right)$ are two synchronously local-almost periodic functions, then, by Definition 3.3, $f, g$ are almost periodic on the same periodic sub-timescales of $\mathbb{T}$. Now, from Theorem 3.22 in [13], the desired conclusion follows immediately. This completes the proof.

Finally, in this paper we consider the following linear dynamic equation on a changingperiodic time scale $\mathbb{T}$ :

$$
x^{\Delta}(t)=A(t) x(t)+f(t)
$$

and its associated homogeneous equation

$$
x^{\Delta}(t)=A(t) x(t)
$$

where $A(t)$ is a local-almost periodic matrix function, and $f(t)$ is a local-almost periodic vector function. Further, we assume that $f(t)$ and $A(t)$ are synchronously local-almost periodic functions.

In the following result we shall use the decomposition theorem of time scales (i.e., Theorem 2.21) to establish the existence and uniqueness of local-almost periodic solutions for the dynamic equation (1).

Theorem 3.5 Let $\mathbb{T}$ be a changing-periodic time scale and $\tau_{t}$ be an index function. If (2) admits an exponential dichotomy on the local part $\mathbb{T}_{\tau_{t}}$ for all $t \in \mathbb{T}$, then (1) has a unique local-almost periodic solution on $\mathbb{T}_{\tau_{t}}$ as follows:

$$
x(t)=\int_{-\infty}^{t} X(t) P_{\tau_{t}} X^{-1}\left(\sigma_{\tau_{t}}(s)\right) \Delta_{\tau_{t}} s-\int_{t}^{+\infty} X(t)\left(I-P_{\tau_{t}}\right) X^{-1}\left(\sigma_{\tau_{t}}(s)\right) f(s) \Delta_{\tau_{t}} s,
$$

where $X(t)$ is the fundamental solution matrix of (2), $P_{\tau_{t}}, I-P_{\tau_{t}}$ are two projections of exponential dichotomy on $\mathbb{T}_{\tau_{t}}, \sigma_{\tau_{t}}$ is the forward jump operator on the periodic sub-timescale $\mathbb{T}_{\tau_{t}}$, $\Delta_{\tau_{t}}$ is the $\Delta$-integral on the periodic sub-timescale $\mathbb{T}_{\tau_{t}}$. 
Proof By Definition 2.9 and Proposition 2.21, we know that $\mathbb{T}=\bigcup_{i=1}^{\infty} \mathbb{T}_{i}, \mathbb{T}_{i}$ is $\omega_{i}$-periodic for each $i \in \mathbb{Z}^{+}$and $\left\{\mathbb{T}_{i}\right\}_{i \in \mathbb{Z}^{+}}$is well connected, so $\mathbb{T}_{\tau_{t}}$ is a periodic sub-timescale. According to Theorem 4.19 in [13], if (2) admits an exponential dichotomy on the periodic subtimescale $\mathbb{T}_{\tau_{t}}$ for all $t \in \mathbb{T}$, then (1) has a unique almost periodic solution on $\mathbb{T}_{\tau_{t}}$ as follows:

$$
x(t)=\int_{-\infty}^{t} X(t) P_{\tau_{t}} X^{-1}\left(\sigma_{\tau_{t}}(s)\right) \Delta_{\tau_{t}} s-\int_{t}^{+\infty} X(t)\left(I-P_{\tau_{t}}\right) X^{-1}\left(\sigma_{\tau_{t}}(s)\right) f(s) \Delta_{\tau_{t}} s .
$$

Hence, Eq. (1) has a unique local-almost periodic solution on the periodic sub-timescale $\mathbb{T}_{\tau_{t}}$. This completes the proof.

Remark 3.6 Note that in (3), s, $t \in \mathbb{T}_{\tau_{t}}$, thus, $\tau_{s}=\tau_{t}$. Hence, (3) can be written as

$$
x(t)=\int_{-\infty}^{t} X(t) P_{\tau_{t}} X^{-1}\left(\sigma_{\tau_{s}}(s)\right) \Delta_{\tau_{s}} s-\int_{t}^{+\infty} X(t)\left(I-P_{\tau_{t}}\right) X^{-1}\left(\sigma_{\tau_{s}}(s)\right) f(s) \Delta_{\tau_{s}} s .
$$

By the above Theorem 3.5, we can get the following corollary.

Corollary 3.7 Let $\mathbb{T}$ be a changing-periodic time scale and $\mathbb{T}_{i}$ be a periodic sub-timescale. If (2) admits an exponential dichotomy on the local part $\mathbb{T}_{i}$ for some $i \in \mathbb{Z}^{+}$, then (1) has a unique local-almost periodic solution on $\mathbb{T}_{i}$ as follows:

$$
x(t)=\int_{-\infty}^{t} X(t) P_{i} X^{-1}\left(\sigma_{i}(s)\right) \Delta_{i} s-\int_{t}^{+\infty} X(t)\left(I-P_{i}\right) X^{-1}\left(\sigma_{i}(s)\right) f(s) \Delta_{i} s,
$$

where $X(t)$ is the fundamental solution matrix of (2), $P_{i}, I-P_{i}$ are two projections of exponential dichotomy on $\mathbb{T}_{i}, \sigma_{i}$ is the forward jump operator on the periodic sub-timescale $\mathbb{T}_{i}$, $\Delta_{i}$ is the $\Delta$-integral on the periodic sub-timescale $\mathbb{T}_{i}$.

In what follows, we will give the concept of combinable-almost periodic functions on changing-periodic time scales by Definition 3.1.

Definition 3.4 Let $\mathbb{T}$ be a changing-periodic time scale. If there exists an $\omega_{i_{0}}$-periodic sub-timescale set $\left\{\mathbb{T}_{i_{0}}\right\}_{i_{0} \in I}$ such that the periods set $\left\{\omega_{i_{0}}\right\}_{i_{0} \in I}$ has a lowest common multiple $\omega$ and $f$ is almost periodic on $\mathbb{T}_{i_{0}}$ for each $i_{0}$, where $I$ is a combinable index number set, then $f$ is called a combinable-almost periodic function on $\mathbb{T}$. In fact, $f$ is almost periodic on the $\omega$-periodic sub-timescale $\bigcup_{i_{0} \in I} \mathbb{T}_{i_{0}}$. Further, if $\bigcup_{i_{0} \in I} \mathbb{T}_{i_{0}}=\mathbb{T}$, then $f$ is called the globally combinable-almost periodic function on $\mathbb{T}$.

The following two corollaries are immediate consequences of Theorem 3.5.

Corollary 3.8 Let $\mathbb{T}$ be a changing-periodic time scale and $f$ be a combinable-almost periodic function on $\mathbb{T}$, and I be a combinable index number set. Then (1) has a unique combinable-almost periodic solution on $\bigcup_{i \in I} \mathbb{T}_{i}$ given by

$$
x(t)=\int_{-\infty}^{t} X(t) P_{c} X^{-1}\left(\sigma_{c}(s)\right) \Delta_{c} s-\int_{t}^{+\infty} X(t)\left(I-P_{c}\right) X^{-1}\left(\sigma_{c}(s)\right) f(s) \Delta_{c} s,
$$

where $X(t)$ is the fundamental solution matrix of (2), $P_{c}, I-P_{c}$ are two projections of exponential dichotomy on $\bigcup_{i \in I} \mathbb{T}_{i}, \sigma_{c}$ is the forward jump operator on the periodic sub-timescale $\bigcup_{i \in I} \mathbb{T}_{i}$, and $\Delta_{c}$ is the $\Delta$-integral on the periodic sub-timescale $\bigcup_{i \in I} \mathbb{T}_{i}$. 
Corollary 3.9 The function $f$ is globally combinable-almost periodic on $\mathbb{T}$ if and only iff is an almost periodic function on the periodic time scale $\mathbb{T}$.

Proof Since $\mathbb{T}_{i}$ is an $\omega_{i}$-periodic sub-timescale, from $\mathbb{T}=\bigcup_{i \in I} \mathbb{T}_{i}$, we find that $\mathbb{T}$ is an $\omega$-periodic time scale, where $\omega$ is a lowest common multiple of $\left\{\omega_{i}\right\}_{i \in I}$ and $I$ is a combinable index number set. Hence, from Definition 3.4 the desired conclusion follows immediately. This completes the proof.

Remark 3.10 From Corollary 3.9 it follows that the concept of almost periodic functions on periodic time scales is equivalent to the concept of globally combinable-almost periodic functions on changing-periodic time scales.

Next, we introduce the concepts of local-almost automorphic functions on changingperiodic time scales.

Definition 3.5 Let $\mathbb{X}$ be a Banach space and $\mathbb{T}$ be a changing-periodic time scale.

(i) Let $f: \mathbb{T} \rightarrow \mathbb{X}$ be a bounded continuous function. We say that $f$ is local-almost automorphic if for every adaption factor sequence $\left\{s_{n}^{\tau}\right\}_{n=1}^{\infty} \subset S_{\tau_{t}} \subset \Pi$, we can extract a subsequence $\left\{\tau_{n}^{\tau}\right\}_{n=1}^{\infty}$ such that

$$
g(t)=\lim _{n \rightarrow \infty} f\left(t+\tau_{n}^{\tau}\right)
$$

is well defined for each $t \in \mathbb{T}$, and

$$
\lim _{n \rightarrow \infty} g\left(t-\tau_{n}^{\tau}\right)=f(t)
$$

for each $t \in \mathbb{T}$. We shall denote by $A A(\mathbb{T}, \mathbb{X})$ the set of all such functions.

(ii) A continuous function $f: \mathbb{T} \times B \rightarrow \mathbb{X}$ is said to be local-almost automorphic if $f(t, x)$ is local-almost automorphic in $t \in \mathbb{T}$ uniformly for all $x \in B$, where $B$ is any bounded subset of $\mathbb{X}$ or $B=\mathbb{X}$. We shall denote by $A A(\mathbb{T} \times \mathbb{X}, \mathbb{X})$ the set of all such functions.

Remark 3.11 Since the changing-periodic time scales include periodic and almost periodic time scales, if $\mathbb{T}$ is a $\tilde{\tau}$-periodic time scale, then $\mathbb{T}_{r}=\emptyset, R_{0}=\emptyset$ and $S_{\tau_{t}}=\{n \tilde{\tau}: n \in \mathbb{Z}\}$. Thus, Definition 3.5 is equivalent to Definition 3.15 in [16]. Further, if $\mathbb{T}$ is an almost periodic time scale, then $\mu$ is bounded, and hence $\mathbb{T}$ is a changing-periodic time scale. Therefore, Definition 3.5 is more general than Definition 45 in [14] because Definition 3.5 covers the almost automorphy on the part $\mathbb{T} \backslash \mathbb{T}_{0}$ of Definition 45 in [14].

Remark 3.12 From Definition 3.5 and the property of $S_{\tau_{t}}$, we can obtain the local almost automorphy on the periodic sub-timescale $\mathbb{T}_{\tau_{t}}$.

In fact, as a consequence of Definition 3.5, we have the following proposition.

Proposition 3.2 Let $\mathbb{T}$ be a changing-periodic time scale. If $f \in C\left(\mathbb{T} \times D, \mathbb{E}^{n}\right)$ is a localalmost automorphic in $t$ uniformly for $x \in D$, then $f \in C\left(\mathbb{T}_{0} \times D, \mathbb{E}^{n}\right)$ is local-almost automorphic in $t$ uniformly for $x \in D$, where $\mathbb{T}_{0}$ is a changing-periodic time scale and $\mathbb{T}_{0} \subset \mathbb{T}$. 
Proof Let $f \in C\left(\mathbb{T} \times D, \mathbb{E}^{n}\right)$ be uniformly local-almost automorphic, then by Definition 3.5, for any adaption factors sequence $\left(\alpha^{\tau}\right)^{\prime} \subset S_{\tau_{t}} \subset \Pi$, there exists a subsequence $\alpha^{\tau} \subset\left(\alpha^{\tau}\right)^{\prime}$ such that $T_{\alpha^{\tau}} f(t, x)=g(t, x)$ and $T_{-\alpha^{\tau}} g(t, x)=f(t, x)$ for each $t \in \mathbb{T}$ uniformly for $x \in S$, where $S$ is any compact set in $D$. Thus it follows that $T_{\alpha^{\tau}} f(t, x)=g(t, x)$ and $T_{-\alpha^{\tau}} g(t, x)=$ $f(t, x)$ for each $t \in \mathbb{T}_{0}$ uniformly for $x \in S$. This completes the proof.

Definition 3.6 Let $f, g \in C\left(\mathbb{T} \times D, \mathbb{E}^{n}\right)$ be uniformly local-almost automorphic and $\mathbb{T}$ be a changing-periodic time scale. We say $f$ and $g$ are synchronously local-almost automorphic if $f, g$ are almost automorphic on the same periodic sub-timescales of $\mathbb{T}$.

Now we assume that in (1) and (2) $A(t)$ is a local-almost automorphic matrix function and $f(t)$ is a local-almost automorphic vector function. Further, we let $f(t)$ and $A(t)$ be synchronously local-almost automorphic functions.

In the following result, as a further application of our decomposition theorem of time scales (i.e., Theorem 2.21), we shall establish the existence and uniqueness of local-almost automorphic solutions for the dynamic equation (1).

Theorem 3.13 Let $\mathbb{T}$ be a changing-periodic time scale and $\tau_{t}$ be an index function. Suppose that $A \in \mathcal{R}\left(\mathbb{T}, \mathbb{R}^{n \times n}\right)$ is almost automorphic and nonsingular on $\mathbb{T}_{\tau_{t}}$ and $\left\{A^{-1}(t)\right\}_{t \in \mathbb{T}_{\tau_{t}}}$, $\left\{\left(I+\mu_{\tau_{t}}(t) A(t)\right)^{-1}\right\}_{t \in \mathbb{T}_{\tau_{t}}}$ are bounded for all $t \in \mathbb{T}$. If (2) admits an exponential dichotomy on the local part $\mathbb{T}_{\tau_{t}}$, then (1) has a unique local-almost automorphic solution on $\mathbb{T}_{\tau_{t}}$ as follows:

$$
x(t)=\int_{-\infty}^{t} X(t) P_{\tau_{t}} X^{-1}\left(\sigma_{\tau_{t}}(s)\right) \Delta_{\tau_{t}} s-\int_{t}^{+\infty} X(t)\left(I-P_{\tau_{t}}\right) X^{-1}\left(\sigma_{\tau_{t}}(s)\right) f(s) \Delta_{\tau_{t}} s,
$$

where $X(t)$ is the fundamental solution matrix of (2), $P_{\tau_{t}}, I-P_{\tau_{t}}$ are two projections of exponential dichotomy on $\mathbb{T}_{\tau_{t}}, \sigma_{\tau_{t}}$ is the forward jump operator on the periodic sub-timescale $\mathbb{T}_{\tau_{t}}, \Delta_{\tau_{t}}$ is the $\Delta$-integral on the periodic sub-timescale $\mathbb{T}_{\tau_{t}}$.

Proof By Definition 2.9 and Proposition 2.21, we know that $\mathbb{T}=\bigcup_{i=1}^{\infty} \mathbb{T}_{i}, \mathbb{T}_{i}$ is $\omega_{i}$-periodic for each $i \in \mathbb{Z}^{+}$and $\left\{\mathbb{T}_{i}\right\}_{i \in \mathbb{Z}^{+}}$is well connected, thus, $\mathbb{T}_{\tau_{t}}$ is a periodic sub-timescale. According to Theorem 5.6 in [16], if (2) admits an exponential dichotomy on the periodic sub-timescale $\mathbb{T}_{\tau_{t}}$ for all $t \in \mathbb{T}$, then (1) has a unique almost automorphic solution on $\mathbb{T}_{\tau_{t}}$ as follows:

$$
x(t)=\int_{-\infty}^{t} X(t) P_{\tau_{t}} X^{-1}\left(\sigma_{\tau_{t}}(s)\right) \Delta_{\tau_{t}} s-\int_{t}^{+\infty} X(t)\left(I-P_{\tau_{t}}\right) X^{-1}\left(\sigma_{\tau_{t}}(s)\right) f(s) \Delta_{\tau_{t}} s .
$$

Hence, Eq. (1) has a unique local-almost automorphic solution on the periodic subtimescale $\mathbb{T}_{\tau_{t}}$. This completes the proof.

Remark 3.14 Note that in (4), s, $t \in \mathbb{T}_{\tau_{t}}$, thus $\tau_{s}=\tau_{t}$. Hence, (4) can also be written as

$$
x(t)=\int_{-\infty}^{t} X(t) P_{\tau_{t}} X^{-1}\left(\sigma_{\tau_{s}}(s)\right) \Delta_{\tau_{s}} s-\int_{t}^{+\infty} X(t)\left(I-P_{\tau_{t}}\right) X^{-1}\left(\sigma_{\tau_{s}}(s)\right) f(s) \Delta_{\tau_{s}} s .
$$

By the above Theorem 3.13, we can get the following corollary. 
Corollary 3.15 Let $\mathbb{T}$ be a changing-periodic time scale and $\mathbb{T}_{i}$ be a periodic subtimescale. Suppose that $A \in \mathcal{R}\left(\mathbb{T}, \mathbb{R}^{n \times n}\right)$ is almost automorphic and nonsingular on $\mathbb{T}_{i}$ and $\left\{A^{-1}(t)\right\}_{t \in \mathbb{T}_{i}},\left\{\left(I+\mu_{i}(t) A(t)\right)^{-1}\right\}_{t \in \mathbb{T}_{i}}$ are bounded for some $i \in \mathbb{Z}^{+}$. If (2) admits an exponential dichotomy on the local part $\mathbb{T}_{i}$, then (1) has a unique local-almost automorphic solution on $\mathbb{T}_{i}$ as follows:

$$
x(t)=\int_{-\infty}^{t} X(t) P_{i} X^{-1}\left(\sigma_{i}(s)\right) \Delta_{i} s-\int_{t}^{+\infty} X(t)\left(I-P_{i}\right) X^{-1}\left(\sigma_{i}(s)\right) f(s) \Delta_{i} s,
$$

where $X(t)$ is the fundamental solution matrix of (2), $P_{i}, I-P_{i}$ are two projections of exponential dichotomy on $\mathbb{T}_{i}, \sigma_{i}$ is the forward jump operator on the periodic sub-timescale $\mathbb{T}_{i}$, $\Delta_{i}$ is the $\Delta$-integral on the periodic sub-timescale $\mathbb{T}_{i}$.

In what follows, we will introduce the concept of combinable-almost automorphic functions on changing-periodic time scales.

Definition 3.7 Let $\mathbb{T}$ be a changing-periodic time scale. If there exists an $\omega_{i_{0}}$-periodic sub-timescale set $\left\{\mathbb{T}_{i_{0}}\right\}_{i_{0} \in I}$ such that the periods set $\left\{\omega_{i_{0}}\right\}_{i_{0} \in I}$ has a lowest common multiple $\omega$ and $f$ is almost automorphic on $\mathbb{T}_{i_{0}}$ for each $i_{0}$, where $I$ is a combinable index number set, then we say $f$ is a combinable-almost automorphic function on $\mathbb{T}$. In fact, then $f$ is almost automorphic on the $\omega$-periodic sub-timescale $\bigcup_{i_{0} \in I} \mathbb{T}_{i_{0}}$. Further, if $\bigcup_{i_{0} \in I} \mathbb{T}_{i_{0}}=\mathbb{T}$, then $f$ is called globally combinable-almost automorphic function on $\mathbb{T}$.

The following two corollaries are immediate consequences of Theorem 3.13.

Corollary 3.16 Let $\mathbb{T}$ be a changing-periodic time scale and $f$ be a combinable-almost automorphic function on $\mathbb{T}$, and I be a combinable index number set. If $A \in \mathcal{R}\left(\mathbb{T}, \mathbb{R}^{n \times n}\right)$ is almost automorphic and nonsingular on $\bigcup_{i \in I} \mathbb{T}_{i}$, and $\left\{A^{-1}(t)\right\}_{t \in \cup_{i \in I} \mathbb{T}_{i}}$ and $\{(I+$ $\left.\left.\mu_{i}(t) A(t)\right)^{-1}\right\}_{t \in \cup_{i \in I} \mathbb{T}_{i}}$ are bounded, then (1) has a unique combinable-almost automorphic solution on $\bigcup_{i \in I} \mathbb{T}_{i}$ given by

$$
x(t)=\int_{-\infty}^{t} X(t) P_{c} X^{-1}\left(\sigma_{c}(s)\right) \Delta_{c} s-\int_{t}^{+\infty} X(t)\left(I-P_{c}\right) X^{-1}\left(\sigma_{c}(s)\right) f(s) \Delta_{c} s
$$

where $X(t)$ is the fundamental solution matrix of (2), $P_{c}, I-P_{c}$ are two projections of exponential dichotomy on $\bigcup_{i \in I} \mathbb{T}_{i}, \sigma_{c}$ is the forward jump operator on the periodic sub-timescale $\bigcup_{i \in I} \mathbb{T}_{i}$, and $\Delta_{c}$ is the $\Delta$-integral on the periodic sub-timescale $\bigcup_{i \in I} \mathbb{T}_{i}$.

Corollary 3.17 The function $f$ is globally combinable-almost automorphic on $\mathbb{T}$ if and only iff is almost automorphic on the periodic time scale $\mathbb{T}$.

Proof Since $\mathbb{T}_{i}$ is an $\omega_{i}$-periodic sub-timescale, from $\mathbb{T}=\bigcup_{i \in I} \mathbb{T}_{i}$ it follows that $\mathbb{T}$ is an $\omega$-periodic time scale, where $\omega$ is a lowest common multiple of $\left\{\omega_{i}\right\}_{i \in I}$ and $I$ is a combinable index number set. Hence, the desired result follows from Definition 3.16. This completes the proof.

Remark 3.18 From Corollary 3.17 it follows that the concept of almost automorphic functions on periodic time scales is equivalent to the concept of globally combinable-almost automorphic functions on changing-periodic time scales. 


\section{Conclusion}

In this work we have introduced a completely new type of time scales - 'changing periodic time scales', and examined some of their properties. The main propose of this kind of time scales is to resolve the problems from an arbitrary time scale to the ones on periodic time scales. Our decomposition theorem of time scales divides an arbitrary time scale into a countable union of periodic time scales. This result not only provides us with a new approach to investigate problems by the known methods on periodic time scales, but also opens up new avenues to study local properties of the functions defined on time scales. It is clearly shown that the changing-periodic time scales are more general than the time scales with bounded graininess function $\mu$, and therefore results obtained on this new type of time scales include all the known results on time scales with bounded graininess function $\mu$. Hence, it is compelling to study problems on changing-periodic time scales. To illustrate the importance of our theory, first we introduce new concepts 'local-almost periodic functions' and 'local-almost automorphic functions' on time scales, and then we use the properties of changing-periodic time scales to establish the localalmost periodicity and local-almost automorphy of solutions of dynamic equations.

Competing interests

The authors declare that they have no competing interests.

\section{Authors' contributions}

All authors contributed equally to the manuscript and typed, read and approved the final manuscript.

\section{Author details}

'Department of Mathematics, Yunnan University, Kunming, Yunnan 650091, People's Republic of China. ${ }^{2}$ Department of Mathematics, Texas A\&M University-Kingsville, 700 University Blvd., Kingsville, TX 78363-8202, USA. ${ }^{3}$ Department of Mathematics, Faculty of Science, King Abdulaziz University, P.O. Box 80203, Jeddah, 21589, Saudi Arabia.

\section{Acknowledgements}

This work is supported by Yunnan University Scientific Research Fund Project in China (No. 2013CG020), Yunnan Province Education Department Scientific Research Fund Project in China (No. 2014Y008), and Yunnan Province Science and Technology Department Applied Basic Research Project in China (No. 2014FB102).

Received: 2 June 2015 Accepted: 3 September 2015 Published online: 17 September 2015

\section{References}

1. Hilger, S: Differential and difference calculus - unified! Nonlinear Anal. 30(5), 2683-2694 (1997)

2. Hilger, S: Generalized theorem of Hartman-Grobman on measure chains. J. Aust. Math. Soc. A 60(2), 157-191 (1996)

3. Agarwal, RP, Bohner, M, O'Regan, D, Peterson, A: Dynamic equations on time scales: a survey. J. Comput. Appl. Math. $141,1-26(2002)$

4. Bohner, M, Peterson, A: Dynamic Equations on Time Scales: An Introduction with Applications. Birkhäuser Boston, Boston (2001)

5. Agarwal, RP, Bohner, M: Basic calculus on time scales and some of its applications. Results Math. 35, 3-22 (1999)

6. Wang, C, Agarwal, RP: Uniformly rd-piecewise almost periodic functions with applications to the analysis of impulsive $\Delta$-dynamic system on time scales. Appl. Math. Comput. 259, 271-292 (2015)

7. Wang, C, Agarwal, RP: Exponential dichotomies of impulsive dynamic systems with applications on time scales. Math. Methods Appl. Sci. (2014). doi:10.1002/mma.3325

8. Wang, C, Li, Y: Weighted pseudo almost automorphic functions with applications to abstract dynamic equations on time scales. Ann. Pol. Math. 108, 225-240 (2013)

9. Wang, C, Agarwal, RP: Weighted piecewise pseudo almost automorphic functions with applications to abstract impulsive $\nabla$-dynamic equations on time scales. Adv. Differ. Equ. 2014, 153 (2014)

10. Wang, C: Almost periodic solutions of impulsive BAM neural networks with variable delays on time scales. Commun. Nonlinear Sci. Numer. Simul. 19, 2828-2842 (2014)

11. Agarwal, RP, Bohner, M, Saker, S: Dynamic Littlewood-type inequalities. Proc. Am. Math. Soc. 143, 667-677 (2015)

12. Kaufmann, ER, Raffoul, YN: Periodic solutions for a neutral nonlinear dynamical equation on a time scale. J. Math. Anal. Appl. 319, 315-325 (2006)

13. Li, Y, Wang, C: Uniformly almost periodic functions and almost periodic solutions to dynamic equations on time scales. Abstr. Appl. Anal. 2011, Article ID 341520 (2011)

14. Wang, C, Agarwal, RP: A further study of almost periodic time scales with some notes and applications. Abstr. Appl. Anal. 2014, Article ID 267384 (2014)

15. Wilansky, A: Topics in Functional Analysis. Lecture Notes in Mathematics, vol. 45. Springer, Berlin (1967)

16. Lizama, C, Mesquita, JG: Almost automorphic solutions of dynamic equations on time scales. J. Funct. Anal. 265 2267-2311 (2013) 